may be especially at risk. However data is scarce and valid comparisons are difficult.

Objective To gauge the prevalence of psychological distress and the level of wellbeing amongst UK Olympic and Paralympic programme athletes.

Design Cross-sectional questionnaire cohort study.

Setting UK Olympic and Paralympic, summer and winter sport athletes.

Patients (or Participants) 394 athletes from 29 sports.

Interventions (or Assessment of Risk Factors) Between October 2018 and June 2019, participants completed the Kessler Psychological Distress Scale (K10) and the World Health Organisation-Five Well-Being Index (WHO5) questionnaires that indicate levels of psychological distress and subjective wellbeing respectively.

Main Outcome Measurements Percentage of athletes reporting low, moderate, high and very high psychological distress. Percentage of athletes reporting high and low wellbeing.

Results $24 \%$ of athletes reported high or very high psychological distress. More Paralympic that Olympic athletes reported high/very high distress ( $27 \%$ vs $22 \%)$. The mean distress score was comparable to age matched population samples. 19\% of athletes reported low psychological wellbeing and this was more common in Paralympic athletes than Olympic athletes (23\% vs $17 \%)$. The mean wellbeing score was comparable to age matched population samples.

Conclusions UK Olympic and Paralympic athletes report psychological distress and wellbeing levels similar to aged matched populations. Paralympic athletes have slightly higher levels of distress and lower wellbeing than Olympic athletes and this may relate to disability specific stressors. Sport programmes should have robust mental health support plans that includes regular athlete screening and commensurate support services with additional specific support for athletes with disabilities.

\section{ACUTE PERIPHERAL FATIGUE INDUCES BRAIN ACTIVITY CHANGES DURING PREDEFINED AND REACTIVE BALANCE TASKS: NEW INSIGHTS CONCERNING THE FATIGUE-INJURY HYPOTHESIS}

${ }^{1}$ Bruno Tassignon, ${ }^{1} \mathrm{~J} o$ Verschueren, ${ }^{1,2}$ Sander De Bock, ${ }^{1}$ Luk Buyse, 1,2 Kevin De Pauw, ${ }^{3}$ Evert Verhagen, ${ }^{1,2}$ Romain Meeusen. 'Human Physiology and Sports Physiotherapy, Brussels, Belgium; ${ }^{2}$ Strategic Research Program Exercise and the Brain in Health and Disease: the added value of Human-Centered Robotics, Vrije Universiteit Brussel, Brussels, Belgium; ${ }^{3}$ Amsterdam Collaboration on Health and Safety in Sports, Department of Public and Occupational Health, Amsterdam Movement Sciences, Amsterdam UMC, Vrije Universiteit Amsterdam, Amsterdam, Netherlands

\subsection{6/bjsports-2021-IOC. 128}

Background An abundance of literature on the acute peripheral fatigue (APF)-injury-hypothesis exists without showing uniformity to support or refute this hypothesis. Previous research demonstrated that a decreased balance ability increases injury risk, and APF affects balance performance. Recently, reactive balance tasks were developed to assess balance performance in a more sport related context. However, it is unknown if APF induces changes in brain activity during predefined and reactive balance tasks and how it relates to injury occurrence.

Objective To study whether (1) APF fatigue alters brain activity during one predefined and one reactive balance task, and (2) performance on these balance tasks.
Design Cross-over design.

Setting Primary clinical setting.

Participant Twenty healthy participants (age $=24 \pm 3$ years; height $=1.8 \pm 0.1 \mathrm{~m}$; weight $=73.2 \pm 11.3 \mathrm{~kg}$ ).

Interventions APF was induced through a 30-second modified Wingate-protocol, while the control task encompassed sitting quietly on the stationary bike for 30s.

Main Outcome Measurements Brain activity was measured through electroencephalography during both balance tasks and computed by means of spectral power analysis. The predefined balance task was the Y-balance test (YBT), while the neurocognitive balance test encompassed the reactive balance test (RBT).

Results Decreased RBT accuracy was observed after APF ( $\mathrm{p}<$ $0.05)$, yet YBT performance and RBT visuomotor reaction time were unaffected. APF induced $\alpha$ - and $\beta$-spectral power increments in the prefrontal, motor and posterior parietal cortex during YBT performance $(\mathrm{p}<0.05)$. For the RBT, an $\alpha$ spectral power increment in the posterior parietal cortex and a $\beta$-spectral power increment in the prefrontal cortex were observed due to APF ( $p<0.05$ ).

Conclusions APF induces different changes in brain activity during predefined and reactive balance tasks. It is likely that different central mechanisms are affected depending on the type of balance task. Further research is needed in an applied setting in order to gain insight in the possible interaction between APF and injury occurrence.

\section{9 MENTAL FATIGUE INTERACTS WITH BRAIN ACTIVITY DURING PREDEFINED AND REACTIVE BALANCE TASKS: IS IT TIME TO ADD MORE CONTEXT TO INJURY PREVENTION SCREENING?}

${ }^{1}$ Bruno Tassignon, 'Jo Verschueren, 'Jeroen Van Cutsem, ${ }^{1,2}$ Kevin De Pauw, ${ }^{1}$ Bart Roelands, ${ }^{3}$ Evert Verhagen, ${ }^{1,2}$ Romain Meeusen. ${ }^{1}$ Human Physiology and Sports Physiotherapy, Brussels, Belgium; ${ }^{2}$ Strategic Research Program Exercise and the Brain in Health and Disease: the added value of Human-Centered Robotics, Vrije Universiteit Brussel, Brussels, Belgium; ${ }^{3}$ Amsterdam Collaboration on Health and Safety in Sports, Department of Public and Occupational Health, Amsterdam Movement Sciences, Amsterdam UMC, Vrije Universiteit Amsterdam, Amsterdam, Netherlands

\subsection{6/bjsports-2021-IOC.129}

Background Athletes indicate that mental fatigue (MF) interferes with maximal performance. MF has also been hypothesized to play an important role in injury occurrence. Clinically applied studies show that MF affects brain activity and decreases endurance, skill and decision-making performance. Nevertheless, no clear insights exist on how MF might affect brain activity during predefined and reactive balance test performance. Gaining insights into this topic could further strengthen or challenge the MF-injury hypothesis.

Objective To study whether (1) MF alters the electrophysiological functioning of the brain during one predefined and one reactive balance task, and (2) performance on these balance tasks.

Design Cross-over design.

Setting Primary clinical setting.

Participants Twelve healthy participants (age $=22 \pm 1$ years; height $=176.9 \pm 8.4 \mathrm{~cm}$; weight $=69.7 \pm 10.4 \mathrm{~kg}$ ).

Interventions MF was induced by a 90-minute Stroop task, while the control task included watching a 90-minute documentary. 